\title{
Establishing material hygrothermal characteristics via long-term monitoring and best-fit numerical models
}

\author{
Mihails Birjukovs ${ }^{1, *}$, Inga Apine ${ }^{2}$, and Andris Jakovics ${ }^{1}$ \\ ${ }^{1}$ University of Latvia, Institute of Numerical Modelling, Riga, Latvia, Jelgavas iela 3, LV-1004 \\ ${ }^{2}$ University of Latvia, Botanical garden, Riga, Latvia, Kandavas iela 2, LV-1083
}

\begin{abstract}
Numerical models of heat and moisture transfer for performance forecast of lightweight insulating assemblies require many inputs. These include exterior climate data (i.e. temperature, relative humidity, solar radiation), interior climate data or standard models, transfer coefficients, correct initial conditions, etc. Most importantly, one needs reliable material models. A material model includes porosity, density, heat capacity, but also non-constant properties, such as thermal conductivity, vapor/liquid water diffusivity, sorption curves. These are, in general, difficult to determine, and material database entries often are incomplete, or simply non-existent. However, if one performs long-term monitoring of temperature and relative humidity dynamics within building envelopes, there is a way to determine hygrothermal curves and properties of the underlying materials. This can be done by performing simulations and finding the set of optimal hygrothermal curves and coefficients such that the experimental data is matched sufficiently well. Despite the appeal, this best-fit model approach is fraught with perils due to many unknowns and must be used carefully. In this article, we demonstrate the application of this method to insulating assemblies for which 6+ years' worth of experimental data is available, and showcase our results obtained using WUFI Pro 6.3 and the derived and verified material models.
\end{abstract}

\section{Introduction}

Standards imposed on buildings such as indoor comfort and energy efficiency are becoming increasingly stricter. At the same time, deadlines for energy consumption reduction for new buildings are due 2020 [1,2]. There is also the matter of risks associated with mould growth $[3,4]$, condensation within wall, ceiling or floor insulation envelopes [3,4] and degradation of materials $[3,5,6]$. This and the great cost of potential mistakes [3] motivates numerical modelling, which allows one to check if the proposed solution performs adequately before committing resources. However, the interplay between heat and moisture transport in building components is quite complex and numerical models require many input parameters and dependencies. These are often unknown a priori or precisely enough. This is a major problem because transport models tend to be very sensitive to inputs and yield misleading results with serious consequences [7].

Clearly, it is imperative to establish and/or verify material models. Therefore, long-term building monitoring projects and test sites have been commissioned, for instance, in Finland [8], Spain [9] and Estonia [10]. In Latvia, roughly six years ago five experimental buildings with different insulating envelopes were constructed at a test site located in Riga. Buildings were equipped with temperature, relative humidity (RH), heat flux and air velocity sensors connected to a data logging system [11,12]. Regardless of the original purpose, the large amount of data accumulated over more than 6 years for these buildings and from the on-site meteorological station enables one to use the experimental data in combination with numerical models for our present study. It must be noted, however, that despite much effort from the local project team over the years ([13-17] and more), many aspects concerning actual initial conditions within buildings and their hygrothermal properties, and thus also long-term behavior, are still unclear. Therefore, adding another layer of verification, samples of materials were taken from building envelopes and analyzed to study the degree of biological contamination by micro-organisms, correlated to RH and temperature dynamics.

With this, we aim to establish hygrothermal models for building materials by finding best-fit numerical models using the accumulated sensor data, verified independently by analyzing biological samples and checking experimental, numerical and laboratory results for consistency using the Sedlbauer mould growth risk model $[18,19]$.

\section{Experiments}

Our test site houses five buildings, codenamed AER, CER, EXP, LOG and PLY, which stand for different materials used for wall envelopes. All five variations have identical shapes, roof and floor assemblies, as well

\footnotetext{
* Corresponding author: mihails.birjukovs@lu.lv
} 
as sensor network layouts (Figure 1). Detailed specifications for each building and sensor placement within are given in [20].

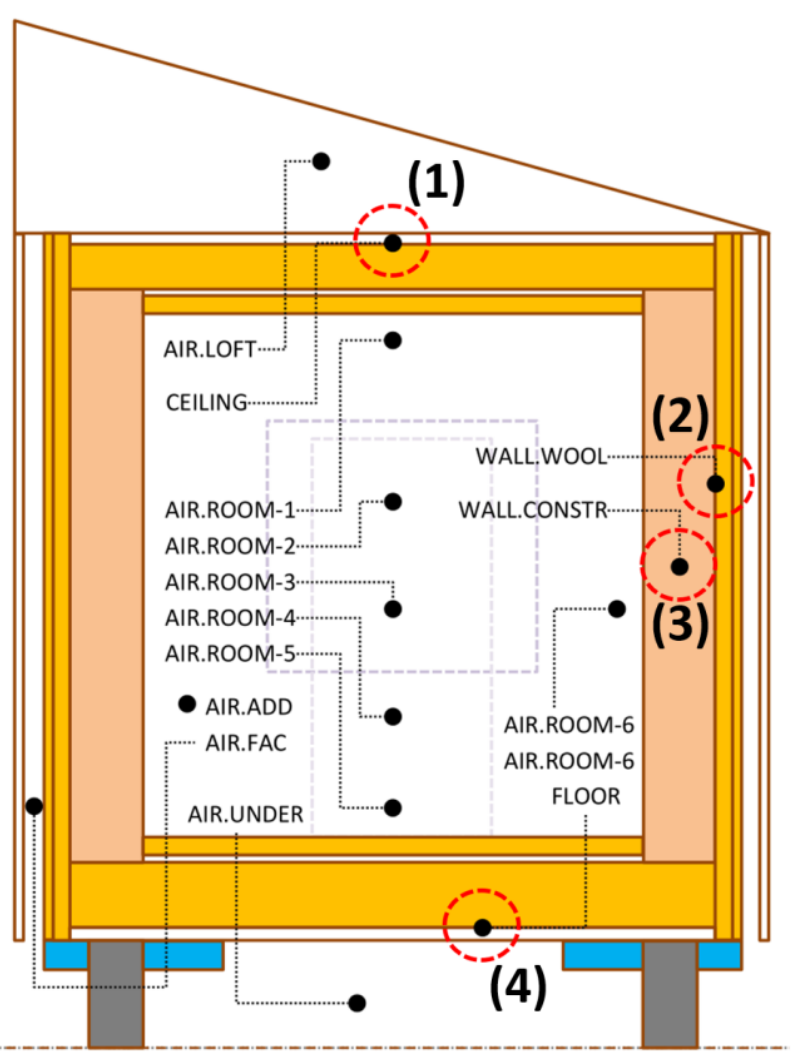

Fig. 1. A layout of the sensor network within a test building where black dots mark sensor locations. There are three types of sensors: air velocity (interior, air gap, attic and outdoors), temperature and RH (at all indicated positions). "ROOM" represents sensors located within building interior, "LOFT" is the attic sensor, "AIR.FAC" is the flow velocity sensor within the air gap of the wall envelope, "CEILING", "WALL" and "FLOOR" are sensors embedded into ceiling, walls and floor, respectively. Sensors relevant for this manuscript are numbered and indicated with red dashed circles.

WUFI (see the next section) is only applicable to parts of assemblies with symmetries that admit 1D models, only data from sensors highlighted in Figure 1 with red dashed circles and wall/floor sensors was used. In this manuscript, we are only going to showcase a part of our results. Specifically, we are going to consider the results for the PLY building and its wall and floor envelopes. The PLY envelopes have the following structure (material, thickness), exterior to interior:

PLY wall: Spruce, $40 \mathrm{~mm} \rightarrow$ Plywood, $6.5 \mathrm{~mm} \rightarrow$ Air gap, $30 \mathrm{~mm} \rightarrow$ Plywood, $20 \mathrm{~mm} \rightarrow$ Stone wool, $200 \mathrm{~mm}$ $\rightarrow$ Plywood, $20 \mathrm{~mm} \rightarrow$ Fibrolite, $75 \mathrm{~mm} \rightarrow$ Cement plaster, $20 \mathrm{~mm}$

PLY floor: Plywood, $21 \mathrm{~mm} \rightarrow$ Vapor barrier, $<1 \mathrm{~mm}$ $\rightarrow$ Stone wool, $200 \mathrm{~mm} \rightarrow$ Plywood, $21 \mathrm{~mm} \rightarrow$ Stone wool, $50 \mathrm{~mm} \rightarrow$ Vapor barrier, $<1 \mathrm{~mm} \rightarrow$ Plywood, 21 $m m$
PLY ceiling: Plywood, $12 \mathrm{~mm} \rightarrow$ Wood wool, $200 \mathrm{~mm}$ $\rightarrow$ Plywood, $4 \mathrm{~mm} \rightarrow$ Stone wool, $50 \mathrm{~mm} \rightarrow$ Vapor barrier, $<1 \mathrm{~mm} \rightarrow$ Plywood, $6.5 \mathrm{~mm}$

Material parameters and that were known due to our own studies are provided in [20], but some are also provided in databases (Fraunhofer-IBP, MASEA, University of Technology Vienna) and those deduced over the course of this study are shown later.

Monitoring of buildings and weather conditions was conducted, at the time of the study, from April 3, 2013 to April 1, 2019., 2019. At the 5-year mark, building envelopes were opened and samples from key locations (including sensors) were taken to the lab for microorganism content assessment.

Data for outdoor climate was used both for interpretation of experimental results and as input for numerical simulations. Details about sensor/outdoor data processing are found in [21]. Micro-organism content analysis steps are given in [22].

\section{Numerical models}

The assemblies were simulated in WUFI Pro 6.3, a finite volume method (FVM) package that solves coupled heat and moisture transport equations under standard building physics assumptions [23]. Outdoor and indoor climate are integrated into Robin boundary conditions for temperature and $\mathrm{RH}$ on the exterior and interior assembly surfaces [23]. Outdoor conditions are given by the processed time series from the meteorological station, while interior conditions are given by ISO 13788 or EN 15026 standards (here referred to as simply ISO or EN) with variable mean temperature and $\mathrm{RH}$ class (moisture load) - the reason is that it is not yet entirely clear to what extent sensor data from within test building rooms are trustworthy and representative of actual inner surface conditions.

While solar radiation is accounted for [23], unfortunately, no records of precipitation dynamics were available, so rain and snow were not considered. Initial conditions for simulations were defined based on sensor measurements and construction/maintenance logs. Note that in some cases initial conditions RH were known only up to an interval. Cloud index is unknown, so a standard coefficient suggested by WUFI for atmospheric counter-radiation is used.

The above parameters and functions must be defined with care, since the transport equations are highly nonlinear [23]. However, this also helps because it means that simulated performance is more likely to significantly differ from experiments if one or more material models are incorrect. Establishing model unknowns is the primary concern of this study.

Hygrothermal transport equations were solved in a 1D approximation. Our estimates based on sensor reading within air gaps in wall envelopes suggest that temperature and $\mathrm{RH}$ that should be close to exterior conditions [21]. This allows one to eliminate the outer weather protection layer from numerical models of wall assemblies. 
The floor insulation assembly is essentially an inverted roof (Figure 1) and is directly exposed to outdoor air. The air gap below is sufficiently wide to disregard grass (periodically trimmed) and shading nullifies the effect of solar radiation. For heat transfer coefficients and details about the numerical aspects, please refer to [21].

\section{Results}

Sensor data and simulation results were processed and compared. Virtual sensors were placed in WUFI Pro 6.3 models in corresponding locations (numbered in Figure 1) and output was processed in the same way as experimental values. Here, several representative results are shown for the PLY building.

Figures $2 \mathrm{a}$ and $2 \mathrm{~b}$ indicate very good agreement for temperature RH dynamics for the PLY ceiling assembly, except the radically different $\mathrm{RH}$ curves are predicted from about the second half of 2018. However, maintenance logs show that these strong deviations correspond to a prolonged series tests for the conditioning system and phase change materials (PCM) that lasted throughout the remainder of the time interval in question. The established (current best) initial and interior conditions for PLY are: initial $\mathrm{RH} \phi_{0}=0.9$ within the wood chip wool, $\phi_{0}=0.6$ elsewhere and EN high moisture load (HML) interior climate. The corresponding material models will be shown later.
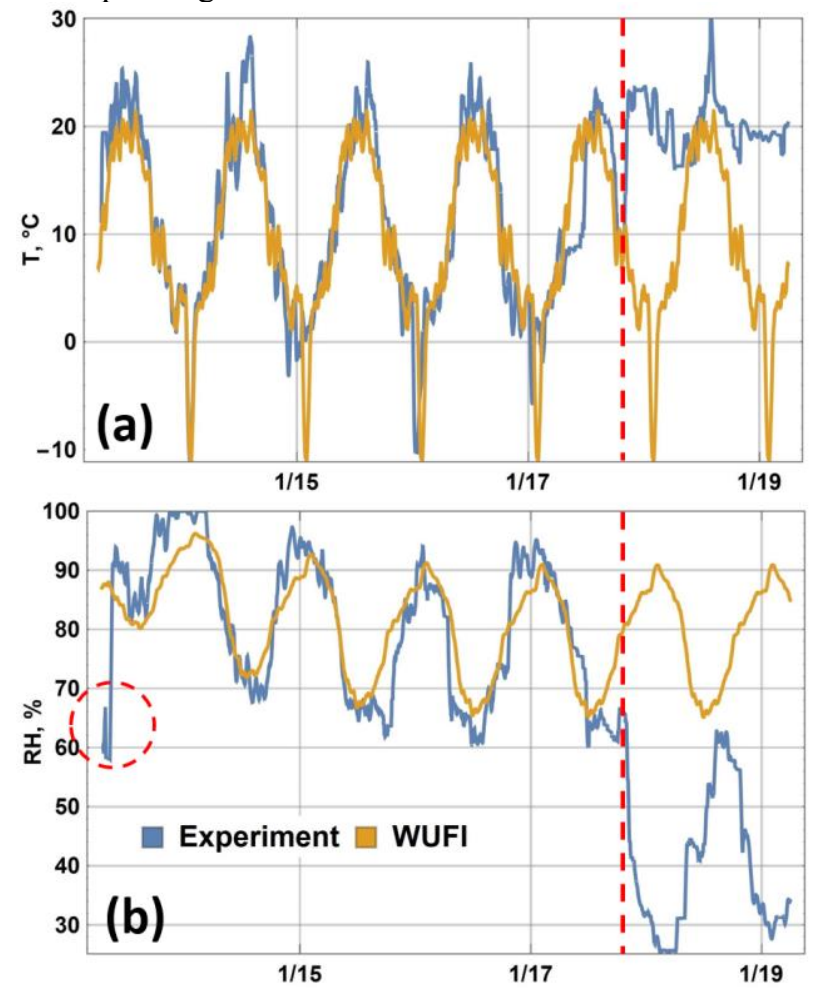

Fig. 2. (a) Temperature and (b) RH dynamics for the PLY ceiling assembly sensor at position 1 (Figure 1): stone wool/vapor barrier boundary. The red dashed circle highlights the region where the sensor temporarily malfunctioned.

Temperature and RH dynamics for the PLY wall assembly are shown in Figure 3 - note the very good temperature curve fit. However, RH time series exhibit systematic overshoots during winter and a deviation near the beginning. Although in Figure $3 \mathrm{~b}$ initial conditions may look different, here $\phi_{0}=0.9$ was indeed set for stone wool and $\phi_{0}=0.6$ elsewhere, as per sensor data, but these initial values were cut off during automatic data filtering. The EN-HML interior climate model was used.
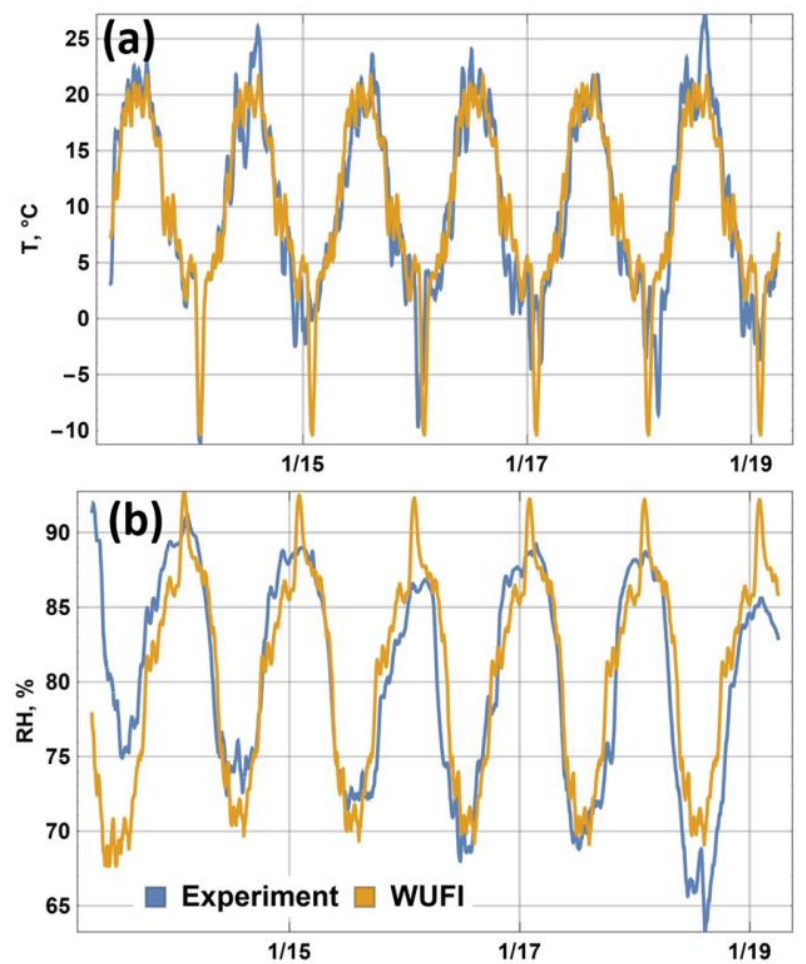

Fig. 3. (a) Temperature and (b) RH dynamics for the PLY wall assembly sensor at position 2 (Figure 1): stone wool/plywood boundary.
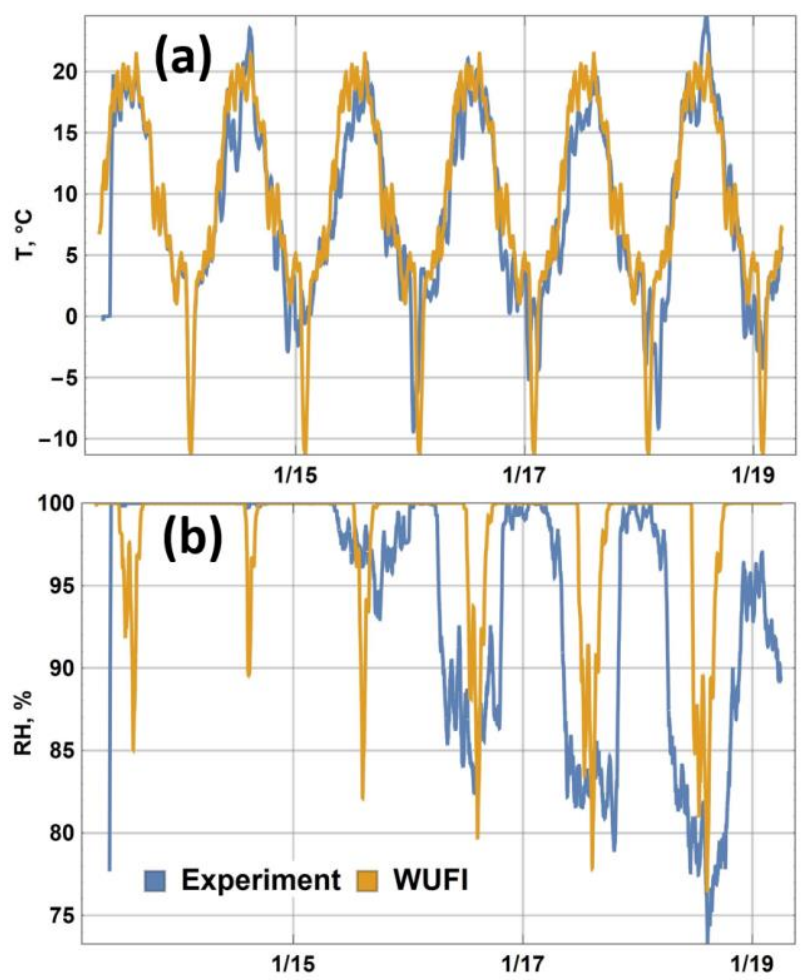

Fig. 4. (a) Temperature and (b) RH dynamics for the PLY floor assembly sensor at position 4 : stone wool/plywood boundary. 
Results for the floor assembly are compared in Figure 4. It must be noted that the vapor barrier here was installed in error and the assembly was inadequately protected from water intake during construction, hence the initial RH values close to 1 . The simulated temperature profiles fit the experiment very well (Figure 4a), but considerable discrepancies are seen for RH dynamics (Figure 4b). While the positions of yearly $\mathrm{RH}$ minima are roughly correct, peak widths clearly do not agree and there is significant RH level mismatch for the first three minima. Since our fits for initial conditions and material models yielded correct results for wall and ceiling assemblies (Figures 2 and 3), the problem is likely due to wrong initial conditions or due to incorrectly specified relative vapor diffusion resistance of the vapor barrier. The diffusion resistance was probably underestimated, which would explain lower inertia (sharper peaks in Figure 4b) and easier initial water exfiltration to the exterior. Here we used $\phi_{0}=1$ globally with EN-HML interior climate.
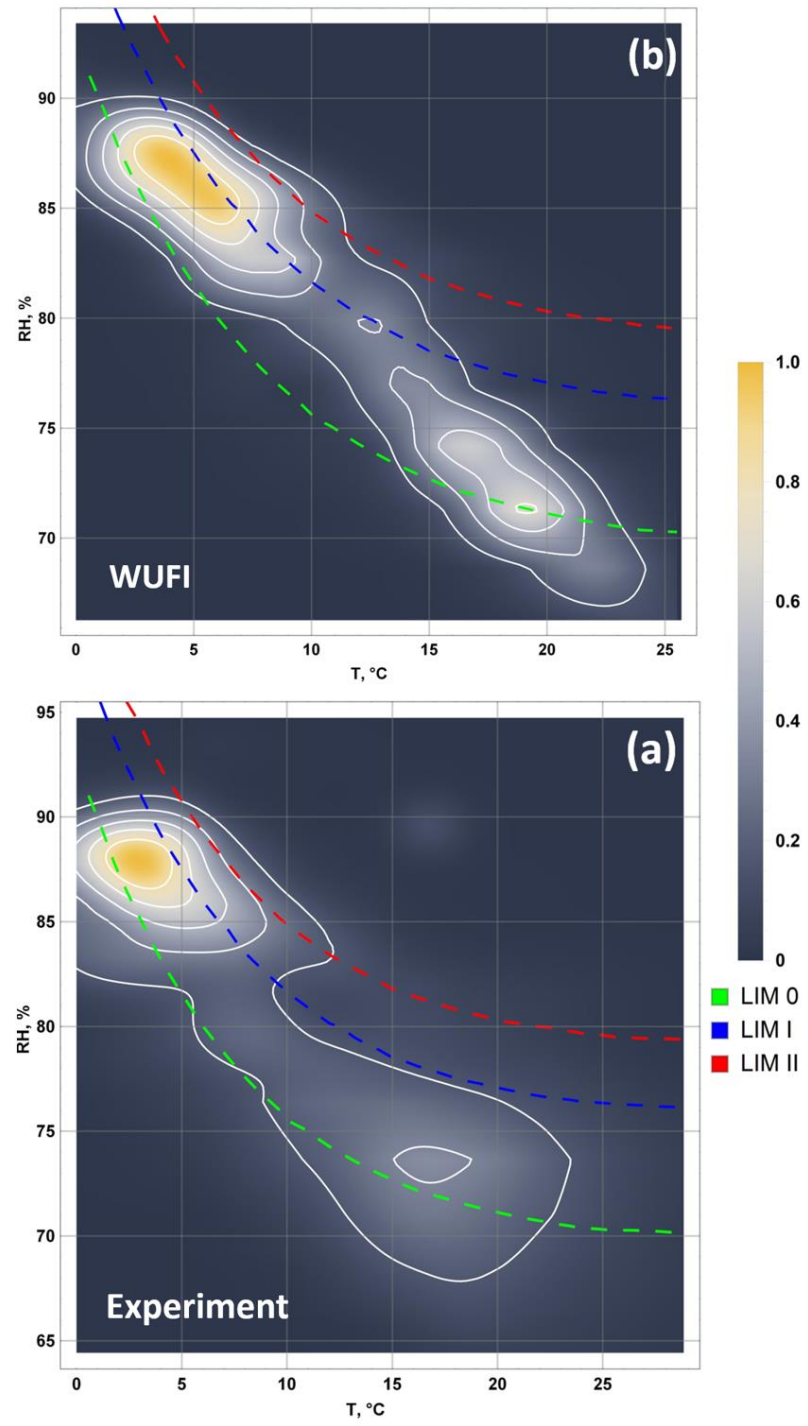

Fig. 5. Mould growth risk assessment from April 3, 2013 to April 1, 2019 for the PLY wall at location 2 (Figure 1), where the sensor placed within the stone wool layer. Temperature and RH pair (event) density is color coded and normalized to maximum. White contours are event density isolines. LIM (lowest isopleth for mould) curves are due to the standard Sedlbauer mould growth risk model. Higher density above the LIM curves implies greater risk of mould growth.

Figure 5 displays the comparison between mould growth risk assessment derived from sensor readings (Figure 5a) and predicted by WUFI (Figure 5b) for the PLY wall at the location of the sensor placed within the stone wool layer. Note the very similar pattern in both cases. Both experimental and simulation data suggest moderate risk for biodegradable materials such as wood wool and minor risk for more resilient materials, such as the stone wool in question, with very little organic content.

A very important point is that this correlates with both visual inspection (no mould was detected) and lab tests conducted for samples taken from the stone wool surface at position 2 (Figure 1). The latter revealed that micro-organism concentrations of $13400 \pm 3454$ $\mathrm{CFU} \cdot \mathrm{g}^{-1}$ (colony forming units per unit sample mass). Compared to $\sim 50 \mathrm{CFU} \cdot \mathrm{g}^{-1}$ for visually clean surfaces and $\sim 55000 \mathrm{CFU} \cdot \mathrm{g}^{-1}$ for mould covered surfaces, this suggests that mould growth risk was indeed only mild to moderate throughout the monitoring period. Interestingly, by far the greatest contributor to mould growth was the mesophilic Cladosporium, which is typically found when $\mathrm{RH} \in[0.8,0.9]$ - this correlates very well with the density maxima seen in Figures $5 \mathrm{a}$ and $5 b$.

The above observations reinforce that, using our best-fit numerical modes, we can now state the validated and/or established material models for the PLY building. For instance, we have validated a model for plywood from the WUFI database. Plywood board: density $\rho=$ $500 \mathrm{~kg} / \mathrm{m}^{3}$, porosity $\mathrm{f}=0.5$, specific heat capacity $\mathrm{c}=$ $1500 \mathrm{~J} /(\mathrm{kg} \cdot \mathrm{K})$, thermal conductivity $\lambda=0.1 \mathrm{~W} /(\mathrm{m} \cdot \mathrm{K})$, relative vapor diffusion resistance $\mu=700$. Hygrothermal characteristics of the plywood board are shown in Figures 6-9 and correspond to an entry in the WUFI database - its elements were not altered during model fitting to check if this database entry is representative of the actual material.

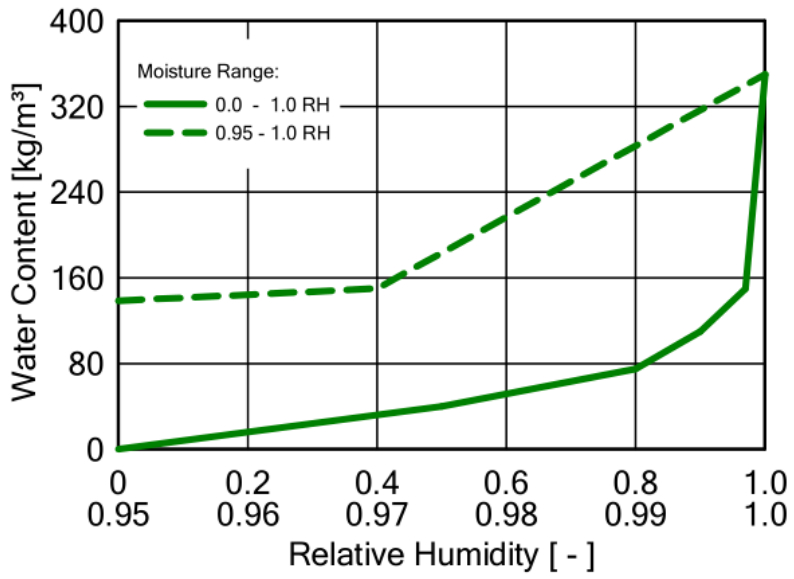

Fig. 6. The sorption curve for the plywood board model. 


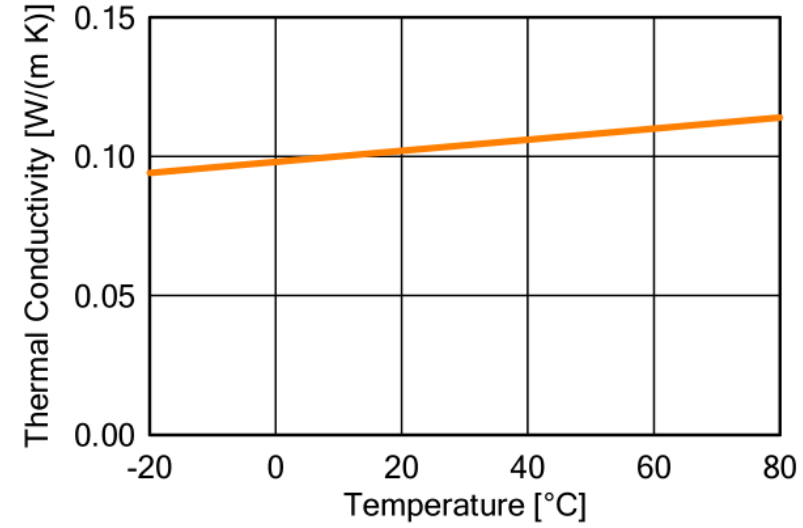

Fig. 7. Thermal conductivity versus temperature for the plywood board model.

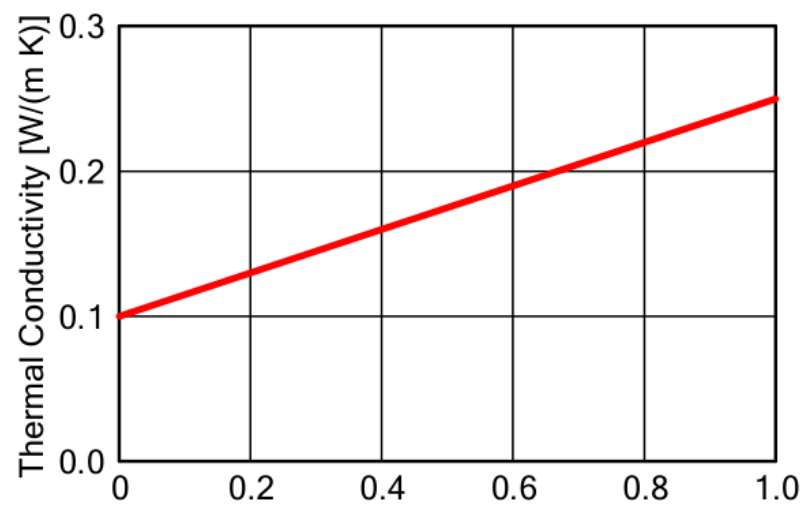

Fig. 8. Thermal conductivity versus RH for the plywood board model.

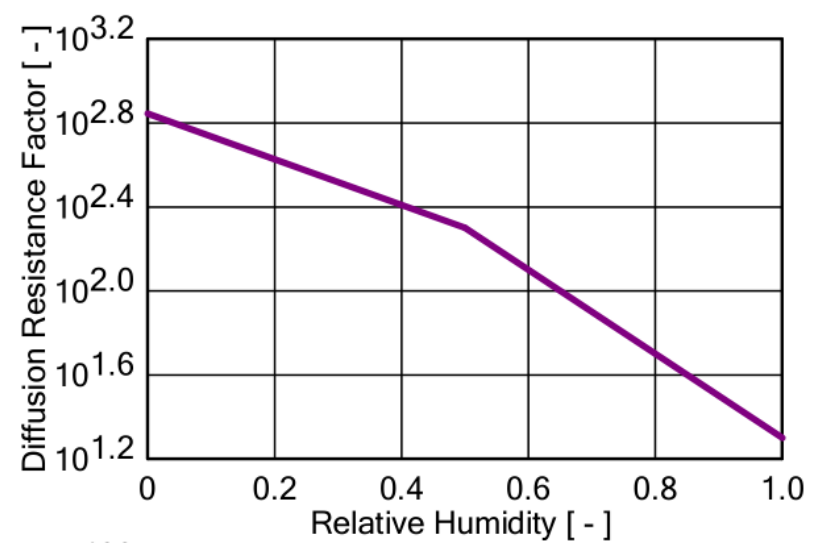

Fig. 9. Relative vapor diffusion resistance versus $\mathrm{RH}$ for the plywood board model.

In addition, we have established a model for PAROC eXtra which, to our knowledge, was not available anywhere. PAROC eXtra (stone wool): $\rho=28 \mathrm{~kg} / \mathrm{m}^{3}, \mathrm{f}=$ 0,$95 ; \mathrm{c}=850 \mathrm{~J} /(\mathrm{kg} \cdot \mathrm{K}) ; \lambda=0,034 \mathrm{~W} /(\mathrm{m} \cdot \mathrm{K}) ; \mu=1$. Hygrothermal characteristics of the PAROC eXtra stone wool slab are shown in Figures 10-12. The relative vapor diffusion resistance for this material was set as constant, which is why no RH dependency curve is given.

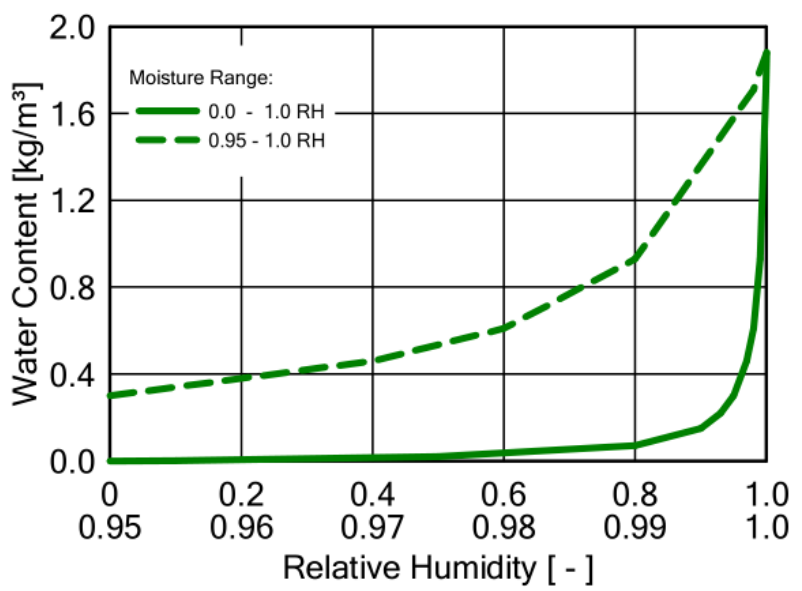

Fig. 10. The sorption curve for the PAROC eXtra model.

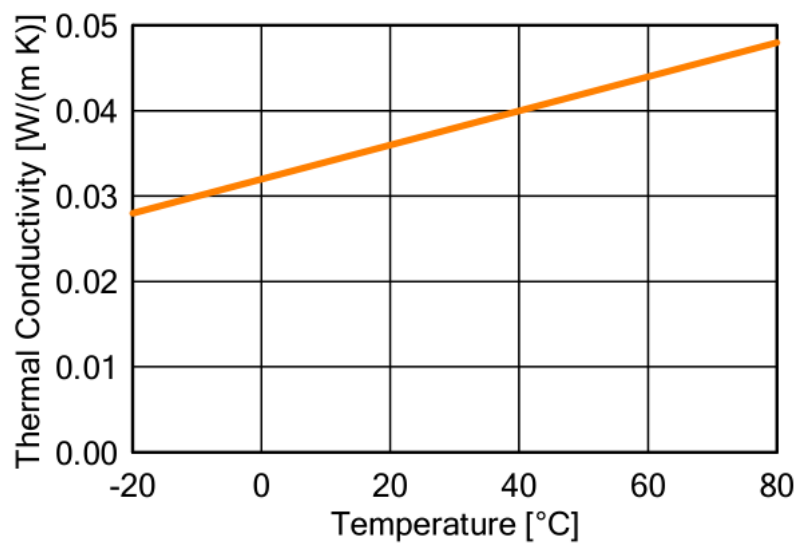

Fig. 11. Thermal conductivity versus temperature for the PAROC eXtra model.

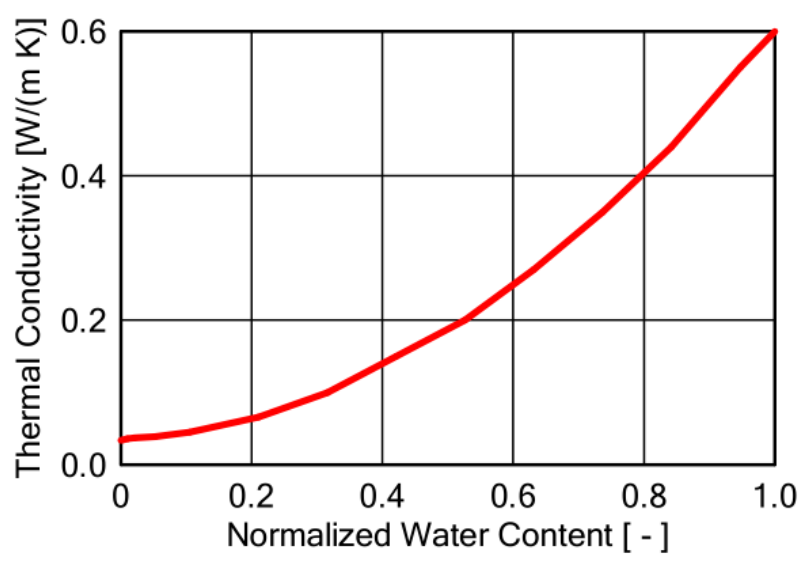

Fig. 12. Thermal conductivity versus RH for the PAROC eXtra model.

\section{Conclusions and outlook}

Numerical models for performance forecast have been established for the insulating envelopes in the PLY building within the University of Latvia test site. Models showed very good agreement with experimental data in terms of RH dynamics, except for the floor envelope, which must be studied further. Temperature time series were in excellent agreement as well.

It was found that both numerical models and processed sensor data correctly predict mould growth 
risks and growth onset (or lack thereof) for wall and ceiling assemblies. The fact that three independent mould growth risk assessment methods (simulations, sensor measurements and lab tests) agree very well leads to the conclusion that our numerical models are on the right track and we can indeed use the best-fit approach to at least roughly determine unknown hygrothermal characteristics of materials.

As a result, we have validated the plywood board material model, which is a part of the WUFI database. In addition, we have determined the sorption and thermal conductivity curves, as well as fixed parameters for PAROC eXtra, which is a rather popular insulation material.

We must to point out that this approach must be tested further and with as much diverse experimental data as possible. The method relies on the strong nonlinearity of the hygrothermal transport equations and thus the sensitivity of numerical models to imprecisions in initial conditions and material characteristics. While our results for PLY seem promising, as do our models for other assemblies from our test site [21], it must be made clear that only carefully analyzing the processes in building envelopes and understanding the function and performance of every material layer can result in usable results. This and three-way verification should help avoid scenarios where very different sets of material characteristics and initial conditions yield similar goodness-of-fit.

This study was conducted with the financial support of European Regional Development Fund, as part of the project "Development, optimization and sustainability evaluation of smart solutions for nearly zero energy buildings in real climate conditions" (1.1.1.1/16/A/192).

\section{References}

1. United Nations Environment Program, Sustainable Buildings and Climate Initiative, ISBN: 987-92-8073064-7 (2009)

2. European Union, Directive 2010/31/EU of the European parliament and council on the energy performance of buildings (2010)

3. N. Othman, M. Jaafar, W. Harun, F. Ibrahim, Procedia Soc. Behav. Sci. 170, 27-36, (2015)

4. J. Holme, S. Geving, J. Jenssen, Moisture and Mould Damage in Norwegian Houses (2008)

5. C. $\mathrm{Xu}, \mathrm{M}$. Mudunuru, K. Nakshatrala, Contin. Mech. Thermodyn. 28 (2015)

6. H. Viitanen, Moisture and Bio-Deterioration Risk of Building Materials and Structures ISBN 978-953307-636-2 (2011)

7. P. Melton, P. Yost, Environmental Building News 23(4), pp 1-10 (2014)

8. J. Vinha, Proceedings of the 8th Symposium on Building Physics in the Nordic Countries NSB2008, pp 1245-1252 (2019)
9. L. F. Cabeza, A. Castell, M. Medrano, I. Martorell, G. Perez, A. Fernandez Energy and Buildings, 42, 630-636 (2010)

10. M. J. Miljan, J. Miljan, Hygrothermal Performance of Timber External Walls Insulated with Natural and Industrial Materials: Sustainable Buildings in Cold Climates, pp 957-967, ISBN 978-3-03000661-7 (2019)

11. I. Beinarts, U. Grunde, A. Jakovics, Electr. Control and Commun. Eng. 7, DOI 10.1515/ecce-20140022 (2014)

12. Greitans M, Grunde U, Jakovics A and Gendelis S 2013, pp 553-556, ISBN 978-1-4799-1419-7

13. J. Ratnieks, A. Jakovics, S. Gendelis, Energy Procedia 132, 441-446 (2017)

14. J. Ratnieks, A. Jakovics, S. Gendelis, Energy Procedia 147, 207-213 (2018)

15. J. Ratnieks, A. Jakovics, S. Gendelis, Energy Procedia 78, 2905-2910 (2015)

16. S. Gendelis, A. Jakovics, J. Ratnieks, L. Bandeniece, IOP Conf. Ser. Mater. Sci. Eng. 251, 012053 (2017)

17. V. Geza, A. Jakovics, S. Gendelis, I. Usilonoks, VIII International Scientific Colloquim "Modelling for Materials Processing", DOI 10.22364/mmp2017.19 (2017)

18. K. Sedlbauer, Prediction of mould fungus formation on the surface of and inside building components, PhD thesis (2001)

19. W. Hofbauer, N. Krueger, K. Breuer, K. Sedlbauer, T. Schoch, Mould resistance assessment of building materials - material specific isopleth-systems for practical application, ISBN 978-87-7877-270-1 (2008)

20. A. Jakovics, S. Gendelis, A. Ozolins, J. Ratnieks, J. Dzerins, J. Klavins, S. Certoks Hygrothermal Performance of Timber External Walls Insulated with Natural and Industrial Materials: Sustainable Buildings in Cold Climates, ISBN 978-9934-51715-0 (2014)

21. M. Birjukovs, I. Apine, A Jakovics, IOP Conf. Ser. Mater. Sci. Eng. 660, (2019)

22. I. Apine, M. Birjukovs, A. Jakovics, Journal of Ecological Engineering 20(11), 256-265 (2019)

23. H. Kunzel, Simultaneous heat and moisture transport in building components. one- and twodimensional calculation using simple parameters, PhD thesis (1995) 